\title{
Log-parabolic spectra and particle acceleration in blazars ${ }^{\star}$
}

\section{The BeppoSAX wide band X-ray spectra of Mkn 501}

\author{
E. Massaro ${ }^{1,2}$, M. Perri $^{3}$, P. Giommi ${ }^{3}$, R. Nesci ${ }^{1}$, and F. Verrecchia ${ }^{3}$ \\ 1 Dipartimento di Fisica, Università La Sapienza, Piazzale A. Moro 2, 00185 Roma, Italy \\ 2 IASF - Sezione di Roma, INAF-CNR, via del Fosso del Cavaliere, 00113 Roma, Italy \\ 3 ASI Science Data Center, ESRIN, 00044 Frascati, Italy
}

Received 27 January 2004 / Accepted 13 April 2004

\begin{abstract}
We present the results of a spectral and temporal study of the complete set of BeppoSAX NFI (11) and WFC (71) observations of the BL Lac object Mkn 501. The WFC 2-28 keV data, reported here for the first time, were collected over a period of about five years, from September 1996 to October 2001. These observations, although not evenly distributed, show that Mkn 501, after going through a very active phase from spring 1997 to early 1999, remained in a low brightness state until late 2001. The data from the LECS, MECS and PDS instruments, covering the wide energy interval $0.1-150 \mathrm{keV}$, have been used to study in detail the spectral variability of the source. We show that the X-ray energy distribution of Mkn 501 is well described by a log-parabolic law in all luminosity states. This model allowed us to obtain good estimates of the SED synchrotron peak energy and of its associated power. The strong spectral variability observed, consisting of strictly correlated changes between the synchrotron peak energy and bolometric flux $\left(E_{\text {peak }} \propto F_{\mathrm{b}}^{\sim 1.4}\right)$, suggests that the main physical changes are not only due to variations of the maximum Lorentz factor of the emitting particles but that other quantities must be varying as well. During the 1997 flare the high energy part of the spectrum of Mkn 501 shows evidence of an excess above the best fit log-parabolic law suggesting the existence of a second emission component that may be responsible for most of the observed variability.
\end{abstract}

Key words. radiation mechanisms: non-thermal - galaxies: active - galaxies: BL Lacertae objects: individual: Mkn 501 $\mathrm{X}$-rays: galaxies

\section{Introduction}

The study of the wide band Spectral Energy Distributions (SEDs) of BL Lac objects (and Blazars in general) has shown that these sources are characterized by a double luminosity peak structure. The peak at lower energies is generally explained by synchrotron radiation from relativistic electrons in a jet closely aligned to the line of sight, while the high frequency bump is produced by inverse Compton scattering. The peak frequency of the first bump ranges from the Infrared-Optical region for the so called Low-energy peaked BL Lac (LBL) objects to the UV-X ray range for the High-energy peaked BL Lac (HBL) (Padovani \& Giommi 1995). The shape of these bumps is characterized in the $\log \left(v F_{v}\right)$ vs. $\log v$ plots by a rather smooth curvature extending through several frequency decades. Analytical models have been proposed to represent these SEDs: a very simple and successful model is a logparabola with only three spectral parameters. In a previous paper (Massaro et al. 2004, hereafter Paper I) we used the

Send offprint requests to: E. Massaro,

e-mail: enrico.massaro@uniroma1.it

* Tables 1 and 2 are only available in electronic form at http://www. edpsciences.org log-parabolic model to fit the BeppoSAX wide band X-ray spectra of the BL Lac object Mkn 421.

In this paper we apply the same spectral analysis to the entire data set of BeppoSAX X-ray observations of Mkn 501 which, like Mkn 421, is an HBL source and has been detected in the $\mathrm{TeV}$ range (Quinn et al. 1996). The X-ray luminosity peak has been observed to be very variable and the energy of the maximum can be as high as about $100 \mathrm{keV}$ (Pian et al. 1998). Mkn $501(z=0.0337)$ has been one of the main targets of multifrequency observational campaigns from ground and space observatories like ASCA, RXTE and BeppoSAX. Among all these observations, those of spring 1997 are particularly interesting because they were taken during a very bright flare when Mkn 501 reached an integral flux in the TeV band of about 10 times that of the Crab Nebula (see, for instance, Aharonian et al. 1999, 2001).

Our spectral analysis is based on all the BeppoSAX observations performed with three of the Narrow Field Instruments (NFIs) on-board this satellite: LECS $(0.1-10 \mathrm{keV})$ (Parmar et al. 1997), MECS (1.4-10 keV) (Boella et al. 1997) and PDS (13-300 keV) (Frontera et al. 1997). Some of these observations have already been analysed by other authors, but with different approaches and applying different spectral laws. 
Pian et al. (1998) presented the data of the 1997 BeppoSAX campaign on Mkn 501 and modelled the spectra with a broken power law. Further analyses of the 1997-1999 observations were presented by Tavecchio et al. (2001), but four observations, performed in June and July 1998, were not included in their work.

In this paper we considered all the 11 NFI BeppoSAX pointed observations of Mkn 501 to verify if a log-parabolic law gives a good description of the synchrotron peak in the SED of this source. Furthermore, we analysed all the serendipitous detections of Mkn 501 with the two Wide Field Cameras (WFCs) on board BeppoSAX (Jager et al. 1997). These two detectors were coded mask cameras operating in the energy range $2-28 \mathrm{keV}$ with a very large field of view $\left(40^{\circ} \times 40^{\circ}\right)$ but with a reduced sensitivity compared to the NFIs. These data are useful to obtain information of the long time evolution of the X-ray luminosity of Mkn 501 over the BeppoSAX lifetime.

In the first part of the paper we present the best fit results of the X-ray observations and discuss the properties of the Spectral Energy Distribution of Mkn 501, also taking into account some simultaneous optical data. In the second part we compare our results with those of other authors and also with our findings on Mkn 421 (Paper I) and discuss some implication of our results on the particle acceleration in the inner jet of this source.

\section{X-ray observations and data reduction}

\subsection{NFI instruments}

BeppoSAX performed a total of 11 pointed observations of Mkn 501: 3 observations in April 1997, 7 in the period from April to July 1998 and the last one in June 1999. The log of all these observations and the net exposure times for the three NFIs considered in our analysis are given in Table 1. Observations were generally concentrated in time windows of several days and the typical MECS exposure time was around $20 \mathrm{ks}$. A longer observation, with interruptions of only few hours, occurred from 1998, April 28 to May 2. In 1999 Mkn 501 was pointed again for a much longer time and the net exposure was about $175 \mathrm{ks}$. We recall that in the observations of April 1997 the MECS operated with all the three detectors, while in the subsequent pointings only two detectors were active.

During the observations the count rate of Mkn 501 was generally stable: the variations, when detectable, did not exceed the $20-30 \%$. For this reason and because of the limited statistics we avoided to segment the data in time or intensity and analysed the entire data set to achieve a better definition of the mean spectral shape.

Standard procedures and selection criteria were applied to the data to avoid the South Atlantic Anomaly, solar, bright Earth and particle contamination using the SAXDAS (v. 2.0.0) package. Data analysis was performed using the software available in the XANADU Package (XIMAGE, XRONOS, XSPEC). The images in the LECS and MECS instruments showed a bright pointlike source: events for spectral analysis were selected in circular regions, centred at the source
Table 3. LECS and MECS energy ranges, reduced $\chi^{2}$ values and inter-calibration parameters for the log-parabolic best fit spectra of Mkn 501.

\begin{tabular}{lccccc}
\hline \hline Date & $\begin{array}{c}\text { LECS } \\
\mathrm{keV}\end{array}$ & $\begin{array}{c}\text { PDS } \\
\mathrm{keV}\end{array}$ & $\chi_{r}^{2} /$ d.o.f. & $f_{\mathrm{LM}}$ & $f_{\mathrm{MP}}$ \\
\hline $1997 / 04 / 07$ & $0.2-2$ & $15-60$ & $1.22 / 112$ & 0.84 & $0.90 \mathrm{f}$ \\
$1997 / 04 / 11$ & $0.12-2$ & $15-130$ & $1.05 / 139$ & 0.85 & $0.83 \mathrm{f}$ \\
$1997 / 04 / 16$ & $0.12-2$ & $15-150$ & $1.01 / 140$ & 0.82 & $0.88 \mathrm{f}$ \\
& & & & & \\
$1998 / 04 / 28-29$ & $0.2-2$ & $15-90$ & $1.10 / 113$ & 0.81 & 0.87 \\
$1998 / 04 / 29-30$ & $0.2-2$ & $15-70$ & $1.02 / 112$ & 0.83 & 0.88 \\
$1998 / 05 / 01-02$ & $0.2-2$ & $15-60$ & $1.09 / 112$ & 0.81 & $0.90 \mathrm{f}$ \\
$1998 / 06 / 20-21$ & $0.13-2$ & $15-30$ & $1.00 / 113$ & 0.81 & $0.90 \mathrm{f}$ \\
$1998 / 06 / 29-30$ & $0.12-2$ & $15-20$ & $1.07 / 112$ & 0.75 & $0.78 \mathrm{f}$ \\
$1998 / 07 / 16-17$ & $0.13-2$ & $15-30$ & $1.06 / 113$ & 0.75 & $0.90 \mathrm{f}$ \\
$1998 / 07 / 25-26$ & $0.13-2$ & $15-45$ & $1.09 / 115$ & 0.78 & 0.91 \\
& & & & & \\
$1999 / 06 / 10-16$ & $0.15-2$ & & $1.18 / 108$ & 0.75 & \\
\hline
\end{tabular}

Note: $\mathrm{f}$ indicates a frozen value.

position, with radii of $4^{\prime}$ and $8^{\prime}$ depending upon the count rate, as indicated by Fiore et al. (1999). Background spectra were taken from blank field archive.

MECS data were always taken in the whole instrumental energy range, while narrower ranges were used for the LECS and PDS. For the LECS we limited the upper bound to $2 \mathrm{keV}$ because above this energy the MECS provided better statistics and to avoid some problems affecting the LECS response matrix, while the lower bound was selected taking into account the signal to noise ratio and the presence of systematic effects, but it was never higher than $0.2 \mathrm{keV}$. We choose the upper bound of the PDS range depending on the source flux and the confusion limit due to the instrumental field of view. As a consequence the useful energy ranges were wider when the source was brighter. In particular for the long observation of 10-16 June 1999 PDS data were not used due to the weakness of Mkn 501. The PDS count rates in the $15-90 \mathrm{keV}$ of each observation are given in Table 1, whereas the energy ranges used for the LECS and PDS data are in Table 3.

In the X-ray spectral fitting we considered the low energy absorption due to the interstellar gas. The Galactic column density was taken equal to $N_{\mathrm{H}}=1.71 \times 10^{20} \mathrm{~cm}^{-2}$, derived from the survey by Dickey \& Lockman (1990) and in very good agreement with the estimate of Stark et al. (1992) adopted by other authors (Pian et al. 1998; Sambruna et al. 2000; Tavecchio et al. 2001). The higher value of $2.87 \times 10^{20} \mathrm{~cm}^{-2}$ was derived by Lamer et al. (1996) from the spectral analysis of ROSAT data; this estimate, however, could be affected by the intrinsic spectral curvature of Mkn 501, not considered by these authors.

\subsection{Wide field cameras}

Mkn 501 was detected by the WFCs on 71 occasions from September 1996 to October 2001. The $\log$ of all these observations is given in Table 2 together with the $2-10 \mathrm{keV}$ flux calculated assuming for the source spectrum a power law 


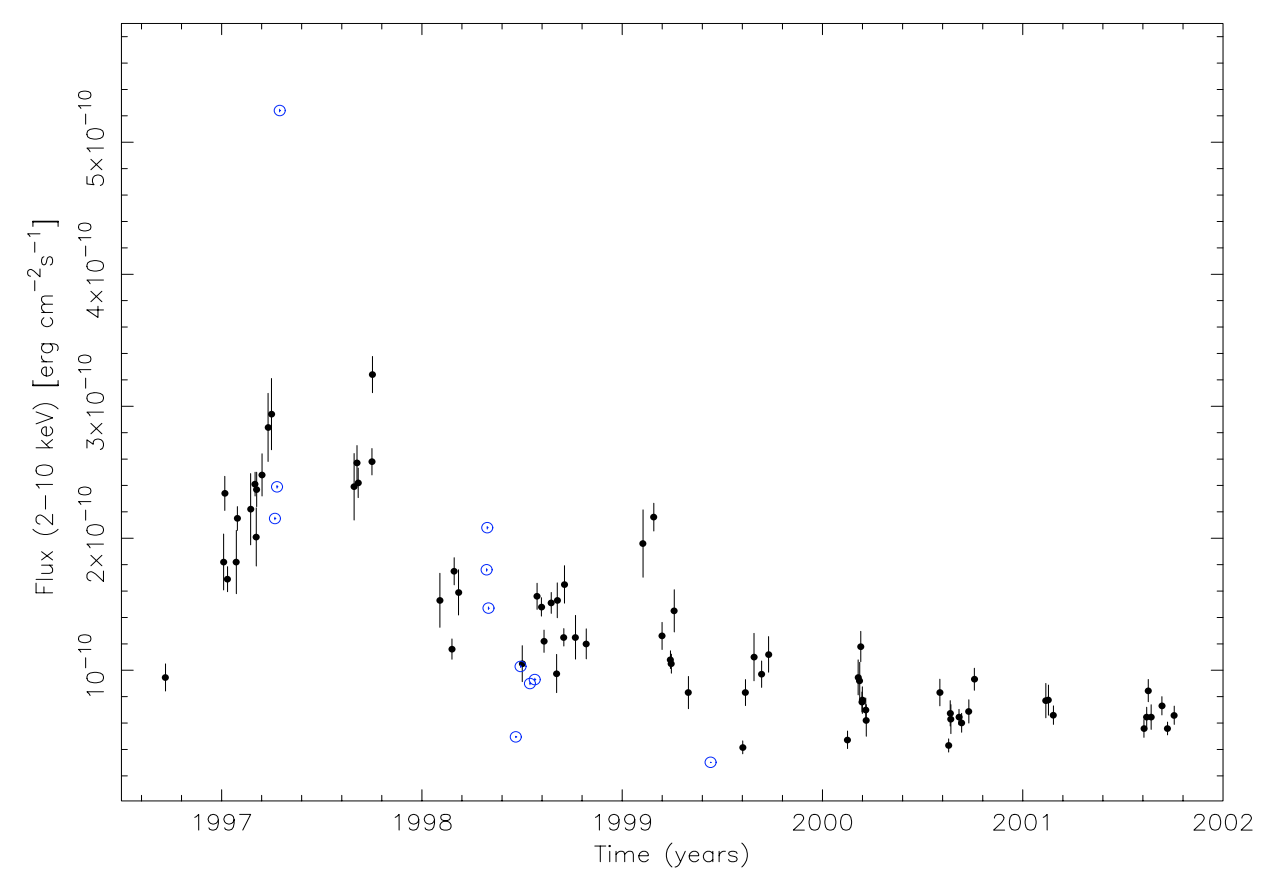

Fig. 1. BeppoSAX 2-10 keV light curve of Mkn 501 observed with WFC (filled circles) and MECS (open circles). Statistical errors of the latter data are smaller than the symbol size.

model with photon index $\Gamma=1.9$. A typical exposure time for the WFC is $30 \mathrm{ks}$ with a few notable exceptions like the observation of 17 August 2001 which lasted over five days.

As for NFIs, standard procedures were applied to WFC data in order to select good time intervals (GTI) for the observations filtering out all unwanted contaminations. Due to the coded mask nature of the instruments, the reconstruction of sky images is achieved through an algorithm that cross-correlates the detector image with the coded mask structure (Fenimore \& Cannon 1978) in an iterative process (Hammersley et al. 1992; in 't Zand 1992).

The subsequent data analysis was performed, as for NFIs instruments, using the XANADU package. The WFC sensitivity depends mainly on the off-axis angle and on the background, so different acceptance criteria on the signal to noise ratio are applied to source identifications depending on the pointing direction and on the off-axis angle. This data reduction technique and the data analysis procedure are implemented in the standard processing used to build the WFC science archive and the source catalog (Piranomonte et al. 2002; Verrecchia et al. in preparation).

The combined MECS and WFC light curve of Mkn 501 in the $2-10 \mathrm{keV}$ band is shown in Fig. 1: the source went through a very active phase from Spring 1997 to early 1999 and afterwards it remained in a low brightness state. Note also that the very high flux measured on 16 April 1997 has never been detected in any other observations. It should be likely considered an exceptional state.

\section{The spectral analysis}

Wide band X-ray spectral distributions of Mkn 501 are remarkably curved, as already pointed out by Tavecchio et al. (2001): the best fits with simple power laws give largely unacceptable $\chi^{2}$ and different analytical models must be considered to describe these spectra. We fitted to the data a power law with an exponential cut-off and generally obtained high reduced $\chi^{2}$, in particular, for the 1997 observations when Mkn 501 was very bright at high energies, the reduced $\chi^{2}$ was never lower than 2 . Tavecchio et al. (2001) modelled the spectra using a continuous combination of two power laws, the same model adopted by Fossati et al. (2000) in the analysis of Mkn 421. No restriction is assumed for the values of the two spectral indices and so it requires four free parameters to be determined. Although this model provides good fits, the use of two (or more) spectral indices at different energies does not allow a direct and simple measure of the SED curvature.

In our analysis we have instead adopted a log-parabolic model for the spectral distribution:

$$
F(E)=K\left(E / E_{1}\right)^{-\left(a+b \log \left(E / E_{1}\right)\right)} \mathrm{ph} /\left(\mathrm{cm}^{2} \mathrm{~s} \mathrm{keV}\right) .
$$

The main properties of this spectral model are summarized in the following (see Paper I for details). We took the reference energy $E_{1}$ fixed to $1 \mathrm{keV}$ and therefore the spectrum is completely determined by the three parameters $K, a$ and $b$. It is possible to define an energy dependent photon index $\Gamma(E)$, given by the log-derivative of Eq. (1):

$\Gamma(E)=a+2 b \log \left(E / E_{1}\right)$

The parameter $a$ is then the photon index at the energy $E_{1}$ and $b$ measures the curvature of the parabola: it is easy to demonstrate that the curvature radius at the parabola vertex is equal to $1 /|2 b|$. A good estimate of $b$ can be obtained only using a sufficiently wide energy range, particularly when its value is small. We define the peak frequency $v_{\mathrm{p}}=E_{\mathrm{p}} / h$, corresponding 
Table 4. Best fit spectral parameters of the log-parabolic model for Mkn 501.

\begin{tabular}{|c|c|c|c|c|c|c|c|}
\hline Date & $a$ & $b$ & K & $E_{\mathrm{p}}(\mathrm{keV})$ & $v_{\mathrm{p}} F\left(v_{\mathrm{p}}\right)^{(2)}$ & $F_{\text {bol }}^{(2)}$ & $F_{2-10 \mathrm{keV}}^{(2)}$ \\
\hline 1997-04-07 & $1.68(0.01)$ & $0.17(0.01)$ & $6.24(0.08) \quad 10^{-2}$ & $8.7(1.3)$ & $1.41(0.05)$ & $9.2(0.4)$ & $2.15(.01)$ \\
\hline 1997-04-11 & $1.64(0.01)$ & $0.12(0.01)$ & $6.09(0.07) \quad 10^{-2}$ & $31.6(9.6)$ & $1.8(0.1)$ & $14.1(1.1)$ & $2.39(.01)$ \\
\hline 1997-04-16 & $1.41(0.01)$ & $0.147(0.007)$ & $9.60(0.1) \quad 10^{-2}$ & $101.6(23.7)$ & $6.0(0.5)$ & $42.2(3.5)$ & $5.24(.01)$ \\
\hline $1998-04-28$ & $1.65(0.02)$ & $0.15(0.02)$ & $4.74(0.08) \quad 10^{-2}$ & $14.7(5.7)$ & $1.2(0.1)$ & $8.4(0.9)$ & $1.76(.01)$ \\
\hline 1998-04-29 & $1.62(0.02)$ & $0.17(0.02)$ & $5.43(0.08) \quad 10^{-2}$ & $13.1(4.3)$ & $1.4(0.1)$ & $9.2(0.9)$ & $2.08(.01)$ \\
\hline 1998-05-01 & $1.71(0.02)$ & $0.24(0.02)$ & $4.77(0.08) \quad 10^{-2}$ & $4.0(0.6)$ & $0.94(0.03)$ & $5.1(0.3)$ & $1.47(.01)$ \\
\hline 1998-06-20 & $1.79(0.02)$ & $0.21(0.02)$ & $1.75(0.04) 10^{-2}$ & $3.2(0.5)$ & $0.32(0.01)$ & $1.9(0.1)$ & $0.495(.005)$ \\
\hline 1998-06-29 & $1.69(0.02)$ & $0.23(0.02)$ & $3.23(0.06) \quad 10^{-2}$ & $4.7(0.8)$ & $0.66(0.03)$ & $3.7(0.2)$ & $1.03(.01)$ \\
\hline $1998-07-16$ & $1.70(0.02)$ & $0.33(0.02)$ & $3.20(0.07) 10^{-2}$ & $2.8(0.3)$ & $0.60(0.02)$ & $2.8(0.1)$ & $0.90(.01)$ \\
\hline $1998-07-25$ & $1.76(0.01)$ & $0.28(0.01)$ & $3.37(0.05) \quad 10^{-2}$ & $2.7(0.1)$ & $0.61(0.01)$ & $3.1(0.1)$ & $0.93(.01)$ \\
\hline $1999-06-10^{(1)}$ & $2.15(0.01)$ & $0.24(0.01)$ & $1.86(0.02) \quad 10^{-2}$ & $0.49(0.03)$ & $0.315(0.004)$ & $1.73(0.04)$ & $0.302(.002)$ \\
\hline
\end{tabular}

Errors are given at 1 sigma for one interesting parameter.

(1) PDS data not included.

(2) In units of $10^{-10} \mathrm{erg} \mathrm{cm}^{-2} \mathrm{~s}^{-1}$.

to the maximum in the $\log v F(v)$ vs. $\log v$ plot, easily computed from the spectral parameters $a$ and $b$, as:

$E_{\mathrm{p}}=E_{1} \times 10^{(2-a) / 2 b}$

and

$v_{\mathrm{p}} F\left(v_{\mathrm{p}}\right)=\left(1.60 \times 10^{-9}\right) K E_{1}^{2} \times 10^{(2-a)^{2} / 4 b} \mathrm{erg} /\left(\mathrm{cm}^{2} \mathrm{~s}\right)$

where the constant is simply the energy conversion factor from keV to erg.

The log-parabolic distribution can be analytically integrated over the entire frequency range to estimate the bolometric flux:

$F_{\text {bol }}=\sqrt{\pi \ln 10} \frac{v_{\mathrm{p}} F\left(v_{\mathrm{p}}\right)}{\sqrt{b}}=2.70 \frac{v_{\mathrm{p}} F\left(v_{\mathrm{p}}\right)}{\sqrt{b}}$.

The log-parabolic law generally provides a good description of the SED in a wide energy interval around the peak. However, at large distances from $E_{\mathrm{p}}$ one can reasonably expect deviations from a perfect parabola. In fact, for low particle energies the acceleration probability may be energy independent originating a single power law spectrum. On the high energy end, the particle spectrum can be limited at the maximum achievable energy producing a rather sharp cutoff in the radiation spectrum. Furthermore, the log-parabola is symmetric with respect to the peak and therefore cannot describe well highly asymmetric distributions. It is easy to modify Eq. (1) to include one more parameter that takes into account of possible asymmetries. However, the spectral analysis of the BeppoSAX observations of Mkn 501 did not require the addition of a new parameter. We then used a log-parabolic law which allowed us to evaluate the mean spectral curvature by means only of the parameter $b$.

As stressed in Paper I, it is important, when working with spectra obtained with two or three NFIs, to use the appropriate inter-calibration factors between MECS and LECS $\left(f_{\mathrm{LM}}\right)$ and PDS $\left(f_{\mathrm{MP}}\right)$. The accurate ground and the in-flight calibrations were used to establish the admissible ranges for these two factors which are: $0.7 \leq f_{\mathrm{LM}} \leq 1.0$ and $0.77 \leq f_{\mathrm{MP}} \leq 0.93$, the latter reduced to $0.86 \pm 0.03$ for sources with a PDS count rate higher than about 2 cts/s (Fiore et al. 1999). The use of intercalibration factors outside these ranges can affect the evaluation of the actual spectral distribution, so these factors cannot be considered as completely free parameters in the fitting procedure or to fix their values to adjust some results. In particular, when a spectrum is intrinsically curved, like in the case of Mkn 501, an improper choice of the inter-calibration factors introduces a bias in the estimation of the spectral parameters. In the analysis of the 1997 observations of Mkn 501 Pian et al. (1998) took $f_{\mathrm{MP}}=0.75$, just outside the lower end of the admissible range. This value was further reduced by $35 \%$ by Krawczynski et al. (2002), but this choice is simply not justified since it is not consistent with the MECS and PDS effective areas. In our evaluations of the spectral parameters, we verified that these two parameters were always inside the nominal ranges: we found $f_{\mathrm{LM}}$ in the range $0.75-0.85$, while on some occasions $f_{\text {MP }}$ was found to be outside the allowed interval and therefore we fixed it to an admissible value. Applying a log-parabolic model, we obtained very satisfactory results for all the observations with well acceptable $\chi^{2}$ values. The best fit values of these two instrumental parameters and the resulting $\chi^{2}$ are given in Table 3 .

The best fit values of the spectral parameters for the logparabolic model are reported in Table 4. For each observation we report the values of $a, b$ and $K$, together with four derived parameters: the peak energy, the SED peak value, the bolometric flux of the considered spectral component and the $2-10 \mathrm{keV}$ flux. Errors correspond to 1 standard deviation for one interesting parameter $\left(\Delta \chi^{2}=1\right)$. 


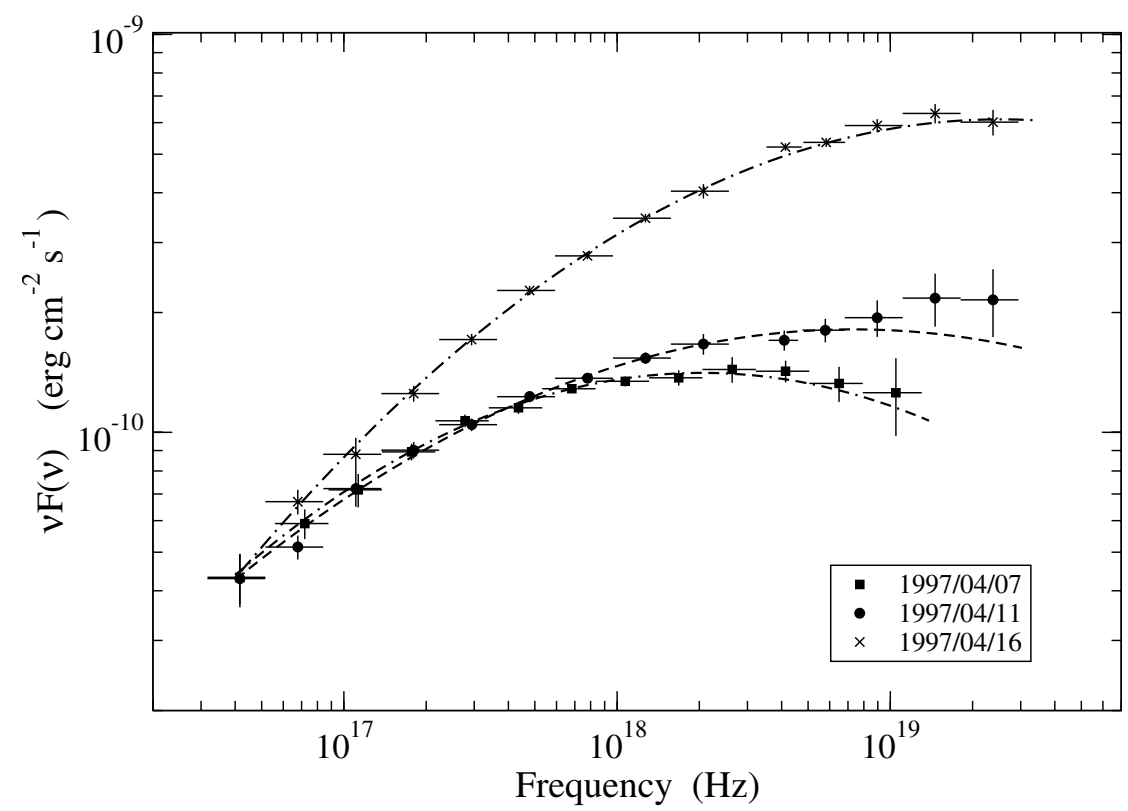

Fig. 2. The X-ray Spectral Energy Distribution of Mkn 501 observed with BeppoSAX in April 1997. The interpolations are the best fits with a log-parabolic law.

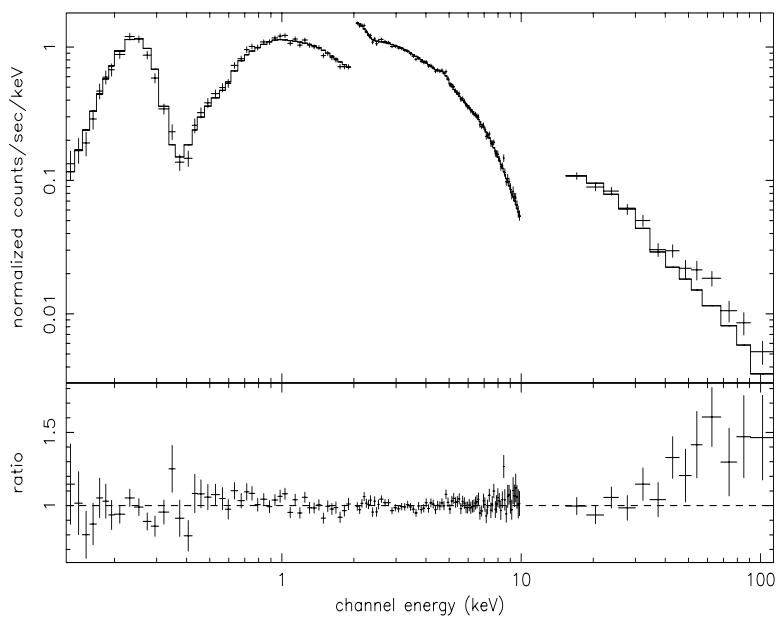

Fig. 3. The best fit spectrum with a log-parabolic law and the residuals of the BeppoSAX observation of Mkn 501 on 11 April 1997. The binning of the data is finer than in Fig. 2. Note the systematic deviation due to a flux excess at energies greater than $30 \mathrm{keV}$.

The three observations of April 1997 were performed during a very active phase of Mkn 501 . The peak energy $E_{\mathrm{p}}$ changed from about $9 \mathrm{keV}$ to $100 \mathrm{keV}$ and correspondingly the $F_{\text {bol }}$ changed by a factor of $\sim 4.5$. The curvature parameter $b$ was generally low, in the range $0.12-0.17$. The SEDs of these observations are shown in Fig. 2. Despite the good $\chi^{2}$, the residuals showed some systematic deviations. In particular, in the spectrum observed on April 11, there is a clear excess at energies above $30 \mathrm{keV}$ and a similar behaviour can also be recognised in the data of April 7. To show in detail how this deviation is significant we plotted the best fit spectrum and residuals of April 11 in Fig. 3: the last seven points in the PDS range are all more than one standard deviation higher than expected. Note also that if the best fit parameters are evaluated limiting the upper energy to $30 \mathrm{keV}$, their values do not change indicating that their estimation is dominated by the low energy data because of their much better statistics. Another important result, very clearly evident in the SEDs of Fig. 2, is that the flux at energies below $0.5 \mathrm{keV}$ remained practically unchanged despite the large variability observed at higher energies. As discussed in Sect. 5, this fact can be relevant to look for a possible interpretation of the spectral evolution of the flare.

A marginal detection (1997, April 4-15) of Mkn 501 with EGRET at energies greater than $\sim 100 \mathrm{MeV}$ is reported by Kataoka et al. (1999) who give the photon flux $F(>100 \mathrm{MeV})=$ $9 \pm 7 \times 10^{-8} \mathrm{ph} \mathrm{cm}^{-2} \mathrm{~s}^{-1}$. We verified that the extrapolation in this range of our log-parabolic fit for the April 16 observation, when the source was at the highest level in the X-ray band, was compatible with this flux. Indeed, using the best fit values of Table 4 , we computed a flux $F(100 \mathrm{MeV})=$ $1.8 \times 10^{-9} \mathrm{ph} \mathrm{cm}^{-2} \mathrm{~s}^{-1} \mathrm{MeV}^{-1}$ corresponding to a power law integral flux of $F(>100 \mathrm{MeV}) \simeq 16 \times 10^{-8} \mathrm{ph} \mathrm{cm}^{-2} \mathrm{~s}^{-1}$. Note that this value, considering that the source during the EGRET pointing was likely weaker than the exceptional flare of April 16 and that its $\gamma$-ray spectrum steeper than our assumption, must be considered as an upper limit. In any case, the April 16 extrapolated value is compatible with the EGRET result within $1 \sigma$.

Observations of Mkn 501 from March to October 1997 at energies higher than $0.25 \mathrm{TeV}$, performed with CAT, are reported by Djannati-Atai et al. (1999), who adopted a logparabolic law to fit the spectra. In particular, in April the source was observed in time windows very close, but not strictly simultaneous, with the BeppoSAX pointings. It showed a behaviour remarkably similar to that in the $\mathrm{X}$ rays with a very strong flare on April 16. The spectral curvature in the $\mathrm{TeV}$ range was always stronger than in the $\mathrm{X}$ rays, in particular the value of $b$ was found greater than 0.4. A similar result is reported by Krennrich et al. (1999), who observed Mkn 501 with the Whipple telescope from February to June 1997. 


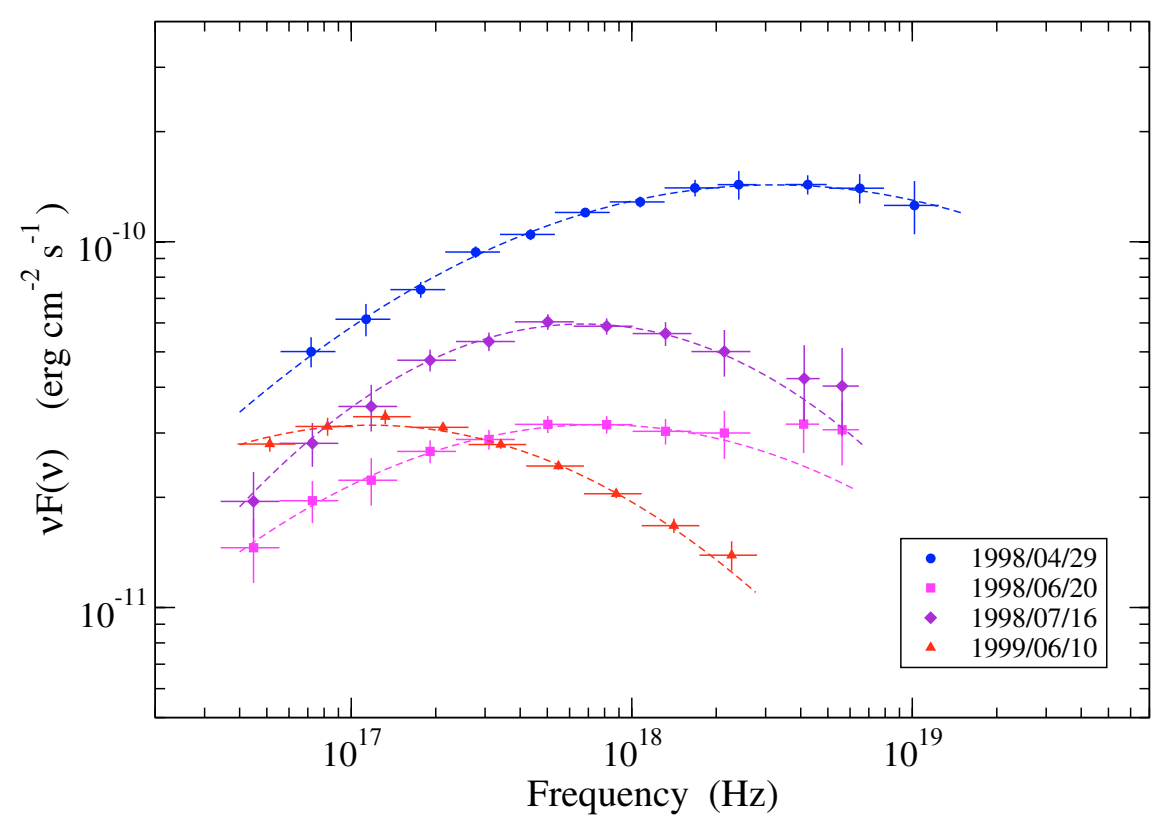

Fig. 4. The X-ray Spectral Energy Distribution of Mkn 501 observed with BeppoSAX in 1998 and 1999. The interpolations are the best fits with a log-parabolic law.

The behaviour of Mkn 501 in the subsequent observations was different from that of 1997. During the long campaign of spring-summer 1998 the source was observed several times: in the two observation of April 1998 it was rather bright with a peak energy around $14 \mathrm{keV}$ and a spectral curvature similar to that measured the previous year. Starting from May 1998, $b$ increased to $0.2-0.3$ and $E_{\mathrm{p}}$ decreased to about 3-4 keV; such state remained rather stable up to the last observation of July 25. The following year Mkn 501 was observed only once when $E_{\mathrm{p}}$ dropped to about $0.5 \mathrm{keV}$, while the curvature was unchanged. Some SEDs of these observations are plotted in Fig. 4. Note that in these observations, contrary to the SEDs of Fig. 2, there is a significant variability of the source flux below $0.5 \mathrm{keV}$.

\section{Optical observations}

Optical observations are useful to verify how and if the $\mathrm{X}$-ray SEDs extend to a much wider frequency interval and whether the log-parabolic model still fits the data even in this range. A direct use of photometric data, especially when obtained with small aperture telescopes, is complicated by the fact that the nuclear emission on Mkn 501 is out-shined by the much brighter host elliptical galaxy. To correct the multiband photometric data for the galaxian component it is necessary to know how much it contributes to the total flux within the used aperture, although other contributions in the optical range from the nuclear environment cannot be excluded.

We performed photometric observations of Mkn 501 in the $B, V$ (Johnson) and $R$, (Cousins) bandpasses on 26 May 1997, about one month after the large outburst in the hard $\mathrm{X}$ rays, 17 and 22 June 1998, close to BeppoSAX pointings, and on 23 June 2000 . We used the $70 \mathrm{~cm}$ reflector telescope of the University of Roma and IASF-CNR located at Monte Porzio and equipped with a back-illuminated CCD camera
Table 5. Optical photometric data of Mkn 501.

\begin{tabular}{lccc}
\hline \hline Date & $B$ & $V$ & $R$ \\
\hline $1997 / 05 / 26$ & & & $13.26 \pm 0.02$ \\
$1998 / 06 / 17$ & & & $13.40 \pm 0.02$ \\
$1998 / 06 / 22$ & $14.83 \pm 0.03$ & $13.89 \pm 0.02$ & $13.32 \pm 0.02$ \\
$2000 / 06 / 23$ & $14.62 \pm 0.02$ & $13.99 \pm 0.02$ & $13.48 \pm 0.02$ \\
\hline
\end{tabular}

(Site 501A). Standard stars were taken from Gonzales-Peres et al. (2001). The magnitudes of Mkn 501, computed with a photometric radius of $4^{\prime \prime}$, are given in Table 5 . The variability is of the order of a tenth of a magnitude and this quite small amplitude can be understood because the flux is dominated by the galaxian contribution which is larger than the nuclear emission. A photometric study of the host galaxy in the $R$ band has been performed by Nilsson et al. (1999), from which one can estimate a magnitude $R_{\text {gal }}=13.90$ for our photometric radius. The magnitudes in the other two bands were estimated assuming typical colours for an elliptical galaxy (Fukugita et al. 1995) and resulted equal to $B_{\text {gal }}=15.47$ and $V_{\text {gal }}=14.51$. Of course, these estimates are affected by systematic errors to be added to the measure uncertainties. Fluxes were then computed using the zero magnitude values from Mead et al. (1990) and the nuclear component was obtained after the subtraction of the host galaxy contribution; the resulting values are $F(R)=5.01$, $F(V)=3.79$ and $F(B)=1.96 \mathrm{mJy}$. We also assumed the extinction $A_{\mathrm{V}}=0.05$ in the direction of Mkn 501 .

The resulting optical-X-ray SED for the observation performed on 20-21 June 1998 is shown in Fig. 5: we plotted the data before and after the subtraction of the host galaxy contribution to illustrate how much this affects the evaluation of the SED. The resulting nuclear spectrum is very steep, although one cannot exclude a further contribution from the 


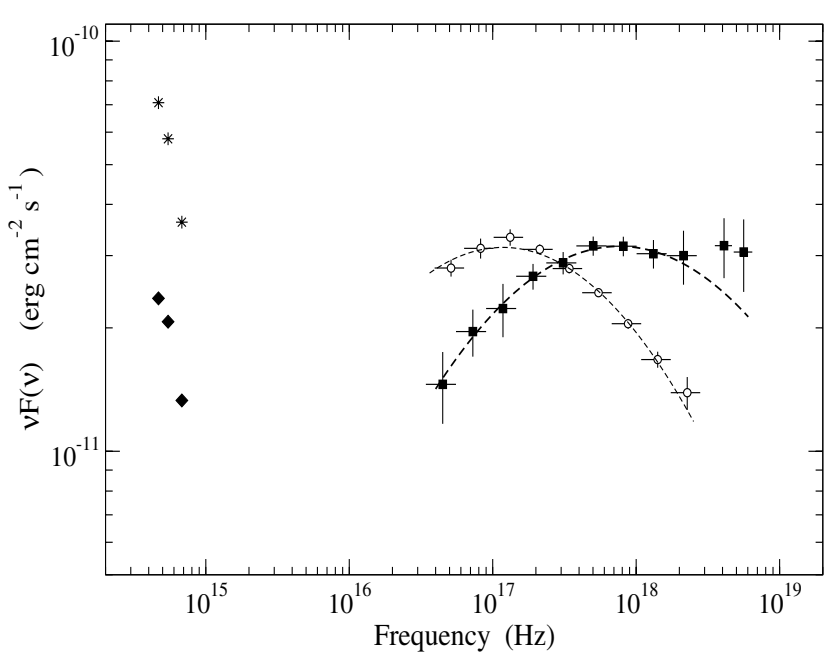

Fig. 5. The optical to X-ray SED of Mkn 501 in June 1998. The X-ray data are those of the June 20-21 (filled squares) and the long-dashed line is the log-parabolic best fit with the parameters given in Table 4. Optical data were obtained during a nearly simultaneous observation (22 June 1998): stars correspond to the measured magnitudes, filled diamonds are the estimated nuclear SED after the subtraction of the host galaxy contribution as described in Sect. 4. For comparison we plotted also the X-ray SED observed in June 1999 (open circles) when Mkn 501 was in a much fainter state.

circumnuclear environment or residual systematic effects. In any case, as for Mkn 421, it is fairly evident that optical data cannot match the low frequency extrapolation of the X-ray spectrum, suggesting the presence of different emission components.

The amplitude of optical variations of Mkn 501 is much smaller than that measured in the $\mathrm{X}$ rays. As discussed above, the amount of nuclear flux largely depends on the subtraction of the galaxian contribution within the considered photometric aperture and therefore the actual luminosity changes of the nuclear emission are poorly known. In any case, to verify if the X-ray emission in the faintest state is compatible with the optical data, we plotted in the Fig. 5 also the X-ray SED of Mkn 501 observed in June 1999 when the SED peak was shifted toward the lower frequencies. We see that also in this case any reasonable extrapolation of the X-ray and optical data cannot match both the flux values and the spectral shape.

\section{Discussion}

Spectral variability in blazars is a very complex issue since it involves rapidly evolving processes that depend on a large number of physical quantities. It is important, therefore, that spectral analysis makes use of models based on simple analytical representations that can be directly related to some physical parameters. In our analysis of the BeppoSAX X-ray observations of Mkn 501, covering more than three decades in energy, we used a log-parabolic spectral model which represents a step in this direction. We have shown that the curved spectra of this source are generally well represented by a law of this type, while a single power law with an exponential cut-off or a broken power law generally do not give equally acceptable best fits.
The most common theoretical approach to produce curved spectra is to consider radiative losses and the escape of high energy electrons from the emitting region: the literature has been summarized in the recent paper by Krawczynski et al. (2002). The intrinsic difficulty is that complex numerical calculations are necessary to solve the transfer equation of the electrons and consequently it is hard to introduce a single parameter to describe the spectral curvature and to find how this is related with the other main physical parameters of the model.

As discussed in Paper I the log-parabolic spectral distribution can be related to the particle acceleration mechanism. We have shown that such a law can be obtained when the acceleration probability is a decreasing function of the particle energy, approximable with a simple power relation, and the curvature parameter $b$ measures this effect. The comparison of the results on Mkn 501 with those on Mkn 421 (Paper I) can be useful to understand how this physical process works in these two sources. As a first point we note that the $b$ values of Mkn 501 are generally smaller than those of Mkn 421: for the latter source we found that $b$ was usually in the range $0.35-0.48$ and decreased to $\sim 0.2$ during the large outburst of May 2000. The values given in Table 4 show that for Mkn $501 b$ was typically $0.18-0.24$ (only in one case it increased to 0.33 ) and during the bright states it decreased to $\sim 0.15$. This result may indicate that the physical conditions in Mkn 501 produce a lower decrease with energy of the particle acceleration probability than in Mkn 421. For instance a larger volume or a higher magnetic field can make more efficient the particle confinement. In this case one can also expect that energetic particle can reach higher energies and this agrees with the higher peak energies observed in Mkn 501. Note, however, that its integrated X-ray luminosity (assumed proportional to $F_{\text {bol }}$ ) is comparable to Mkn 421: in particular in the brightest states it was higher only by a factor of about 2 .

As mentioned in Sect. 3 spectral curvature is also well evident in the TeV range, where Djannati-Atai et al. (1999) and Krennrich et al. (1999) reported for Mkn 501 values of $b$ in excess of 0.4 . Furthermore, the spectrum of Mkn 421, observed by Whipple in May 1996, was characterised by a $b$ value of $0.28 \pm 0.09$, significantly less curved than Mkn 501. X-ray observations of Mkn 421 in May 1996 performed by ASCA showed that the source had a high flaring activity and reached a $2-10 \mathrm{keV}$ flux of $\sim 4 \times 10^{-10} \mathrm{erg} \mathrm{cm}^{-2} \mathrm{~s}^{-1}$ (Kataoka et al. 1999), comparable to that measured in spring 2000 (see Paper I). Unfortunately, measures of $b$ for that period are not available, but if the SED was similar to that observed with BeppoSAX in May 2000 a value of $b$ of $\sim 0.18-0.25$ can be assumed. Under this assumption we found that the X-ray and TeV spectral curvatures of Mkn 421 can be rather similar and thus these emission can be related to the same electron population. The case of Mkn 501 looks different because in the $\mathrm{X}$ rays $b$ values as high as that at $\mathrm{TeV}$ energies has never been measured. From Table 4 we see that the highest curvature $(b \simeq 0.3)$ was found in 1998 when the source was rather faint, and in 1997 it was much brighter for a period lasting several months. We do not know if these differences of the spectral curvature are genuine or due to the superposition of multi-epoch $\mathrm{TeV}$ data. The more pronounced curvature of the $\mathrm{TeV}$ spectra could be produced 


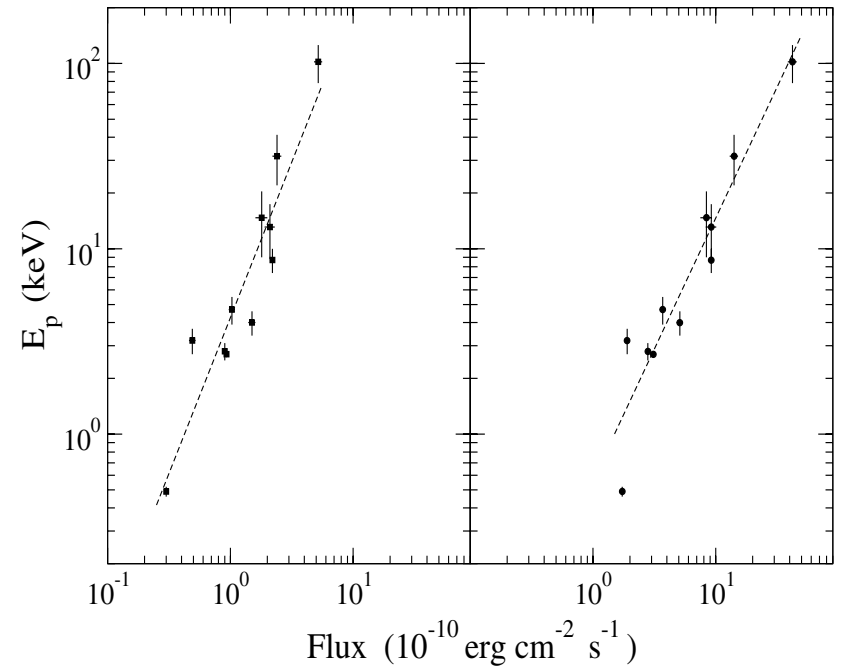

Fig. 6. The correlations between the peak energy of the SEDs and the flux in the $2-10 \mathrm{keV}$ (left panel) and the bolometric flux (right panel) estimated from Eq. (5). Dotted lines are the power law best fits.

by several processes like pair production by energetic photons against the infrared radiation in the intergalactic space or the inverse Compton scattering in the Klein-Nishina regime. In a further paper (Massaro et al. - Paper III, in preparation) we will investigate, by means of a numerical code, the curvature properties of the synchrotron and inverse Compton emission from an electron population with a log-parabolic energy distribution.

The log-parabolic model gives us also the possibility of investigating the correlation between some spectral parameters like the peak energy and the flux. Tavecchio et al. (2001) already searched for this correlation but their result was not very stringent because they found many lower limits on $E_{\mathrm{p}}$ and therefore were only able to conclude that their analysis suggested a relation $E_{\mathrm{p}} \propto F^{n}$ with $n \simeq 2$. In Fig. 6 we plotted the same relation using the best fit values given in Table 4: we used both the flux in the $2-10 \mathrm{keV}$ band and the bolometric flux and found very good correlations with $E_{\mathrm{p}}$. The linear correlation coefficients of $\log E_{\mathrm{p}}$ with $\log F_{2-10 \mathrm{keV}}$ and $\log F_{\text {bol }}$ are 0.937 and 0.948 , respectively, confirming the existence of power relationships. The resulting exponents of these two laws are $1.68 \pm 0.21$ and $1.41 \pm 0.16$, in particular the latter significantly smaller than 2 . According to Tavecchio et al. (2001) the quadratic dependence of the peak energy on the luminosity can be accounted by a simple scenario in which only the maximum Lorentz factor of the emitting electron varies whereas the other physical quantities remain almost constant. Note that in Fig. 6, right panel, there is a point largely out of the correlation: it corresponds to the long 1999 observation, in which the peak energy was very low while the bolometric flux was practically equal to that of 20 June 1998, as also shown by the SEDs in Fig. 4. This can be an indication that the physical conditions in the nucleus of Mkn 501 at that epoch were really very different from those of the preceding observations. Excluding this point, the correlation coefficient improves to 0.963 and the value of $n$ changes to $1.25 \pm 0.12$, even smaller than 2 . We interpret this result as an indication that not only the maximum Lorentz factor changed but other quantities should have varied as well.
The X-ray and TeV emission of Mkn 501 was explained by Tavecchio et al. (2001) with a one-zone SSC model in which the X-ray SED changes are produced by a component with a stable emission below a few $\mathrm{keV}$ and a variable high energy cutoff. Krawczynski et al. (2002), however, found that a onecomponent model fails to fit both the SED and the light curves at different energies from $\mathrm{keV}$ to $\mathrm{TeV}$. These authors obtained better fits considering a two-component model, one of which in the spectral range 3-25 keV, comparable to our LE component (see below). However, they did not show details of the $\mathrm{X}$-ray SEDs and it is not possible to verify if the adopted power law distribution can fit the spectral curvature below $10 \mathrm{keV}$.

We already pointed out in Paper I that the SED of Mkn 421 can be explained by means of the simultaneous presence of different emission components. Likely, the same holds for Mkn 501. Suggestive indications for the presence of more than one component in Mkn 501 are derived from the spectral dynamics during the flare of April 1997. As noted in Sect. 4 the flux at energies below $\sim 0.5 \mathrm{keV}$ remained very stable while that above $\sim 30 \mathrm{keV}$ changed by about an order of magnitude. In particular on April 11 we found evidence for a high energy excess with respect to the log-parabolic best fit. Furthermore, the optical spectrum is generally steep and does not match the low energy extrapolation of the X-ray spectrum. A better description of the observed spectra can be obtained adding a second emission component: in the X-ray range one component (LE) dominates at lower energies and its peak energy in the SED lies between 0.5 and $\sim 5 \mathrm{keV}$, while a second one (HE), responsible for the emission in the hard $\mathrm{X}$ rays, peaks at energies higher than $10 \mathrm{keV}$ and likely up to $\sim 150 \mathrm{keV}$ and can show large variations on short time scales. We assume that the physical mechanism for the particle acceleration and emission are basically the same and therefore the spectra of both components are given by log-parabolic laws. This does not mean that six parameters (instead of three) are necessary to fit the data. In fact, a best fit of the data using two log-parabolas is poorly determined and acceptable solutions are obtained in wide ranges of the parameter space. We then tried to reproduce the observed spectral shape constraining the model with some assumptions. We assumed that the spectra of both components are described by log-parabolic laws and choose the parameter of the LE component approximately equal to those of April 7 ( $a=1.68$, $b=0.18, K=5.7 \times 10^{-2}$, the corresponding SED is the dotted line in Fig. 7) allowing a moderate variability of $a$ to 1.60 and of $K$ to $6.9 \times 10^{-2}$ on April 16. The parameters of the HE component were established to match the sum of the components and the data: on April 11 the values of $a$ and $b$ were 0.50 and 0.35 while on April 7 and 16 the were 0.85 and 0.35 , respectively; the normalisation $K$ varied between $0.95 \times 10^{-3}$ and $23 \times 10^{-3}$. The HE components and the total SEDs are plotted in Fig. 7: the agreement is fully satisfactory and the excess above $30 \mathrm{keV}$ is well reproduced. With these values of $a$ and $b$ the HE peak energy varied between 83 and $140 \mathrm{keV}$. The actual spectral shape of the HE component at energies lower than a $\sim 1 \mathrm{keV}$, for instance a power law instead of a log-parabola, is not important because its contribution to the total flux is about an order of magnitude smaller than the LE component. The $\mathrm{X}$-ray spectral changes observed are therefore mainly produced 


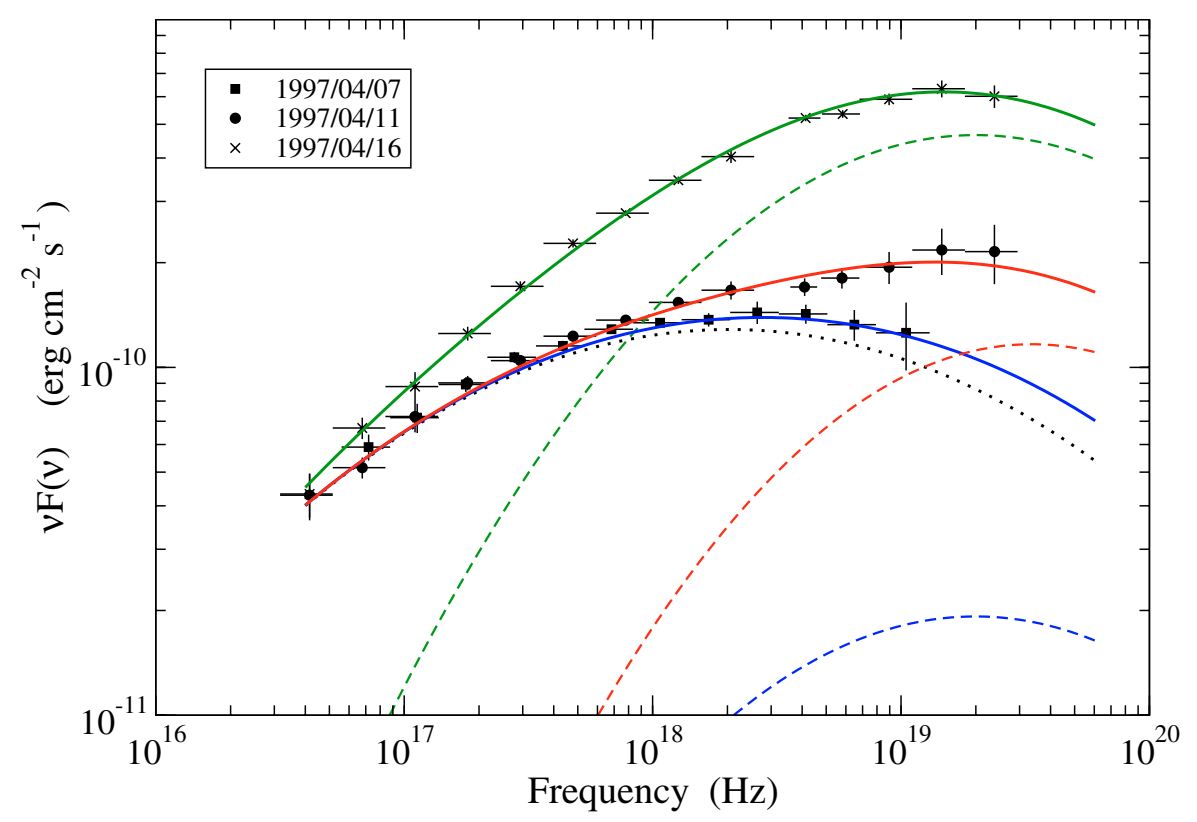

Fig. 7. A two model component of the X-ray flare of Mkn 501 observed in April 1997. The nearly constant component is the dotted line, while three states of the variable component are represented by the dashed lines. Total SEDs are the thick solid lines modelling the observed data.

by a variation of the number of emitting particles of both components: for the LE component the particle number increased of about $20 \%$, while for the HE it increased by a factor of $\sim 25$. Note that this two-component model does not require low $b$ values as those found on April 11 and 16: the smooth SEDs with reduced curvature are obtained by adding components with curvatures similar to those observed in 1998 when Mkn 501 was less bright. Furthermore, the similar $b$ values can be considered as an indication that the particle acceleration does not occur in very different physical conditions. As a final remark we note that these two component model does not affect the correlations of Fig. 6 because it changes only the two highest flux points. The HE component assumed by us does not follow this correlation being its peak energy practically constant despite the large flux change. However this component can be present only during the exceptional flare of April 1997 and thus not represent the typical behaviour of the source.

Acknowledgements. The CNR Institutes and the BeppoSAX Science Data Center are financially supported by the Italian Space Agency (ASI) in the framework of the BeppoSAX mission. Part of this work was performed with the financial support Italian MIUR (Ministero dell' Istruzione Universitá e Ricerca) under the grant Cofin 2001/028773.

\section{References}

Aharonian, F., Akhperjanian, A. G., Barrio, J. A., et al. 1999, A\&A, 349,11

Aharonian, F., Akhperjanian, A. G., Barrio, J. A., et al. 2001, A\&A, 366,62

Boella, G., Chiappetti, L., Conti, G., et al. 1997b, A\&AS, 122, 327

Dickey, J. M., \& Lockman, F. J. 1990, ARA\&A, 28, 215

Djannati-Atai, A., Piron, F., Barrau, A., et al. 1999, A\&A, 350, 17

Elvis, M., Wilkes, B. J., McDowell, J. C., et al. 1994, ApJS, 95, 1

Fenimore, E. E., \& Cannon, T. M. 1978, Appl. Opt., 17, 337
Fiore, F., Guainazzi, M., \& Grandi, P. 1999, Cookbook for BeppoSAX NFI Spectral Analysis (http://www.sdc.asi.it/software) Fossati, G., Celotti, A., Chiaberge, M., et al. 2000, ApJ, 541, 166 Frontera, F., Costa, E., dal Fiume, D., et al. 1997, A\&AS, 122, 357 Fukugita, M., Shimasaku, K., \& Ichikawa, T. 1995, PASP, 107, 945

Gonzales-Peres, J. N., Kidger, M. R., \& Martin-Luis, F. 2001, AJ, 122, 2055

Hammersley, A., Ponman, T., \& Skinner, G. K. 1992, Nuc. Instr. Meth. Phys. Res. A, 311, 585

Inoue, S., \& Takahara, F. 1996, ApJ, 97, 1

Jager, R., Mels, W., Brinkman, A., et al. 1997, A\&AS, 125, 557

Kataoka, J., Mattox, J. R., Quinn, J., et al. 1999, ApJ, 514, 138

Krawczynski, H., Coppi, P. S., \& Aharonian, F. 2002, MNRAS, 336, 721

Krennrich, F., Biller, S. D., Bond, I. H., et al. 1999, ApJ, 511, 149

Lamer, G., Brunner, H., \& Staubert, R. 1996, A\&A, 320, 19

Landau, R., Golisch, B., Jones. T. J., et al. 1986, ApJ, 308, 78

Lockman, F. J., \& Savage, B. D. 1995, ApJS, 97, 1

Massaro, E., Perri, M., Giommi, P., \& Nesci, R. 2004, A\&A, 413, 489 (Paper I)

Mead, A. R. G., Ballard, K. R., Brand, P. W. J. L., et al. 1990, A\&AS, 83, 183

Nilsson, K., Pursimo, T., Takalo, L. O., et al. 1999, PASP, 111, 1223

Padovani, P., \& Giommi, P. 1995, ApJ, 111, 222

Parmar, A. N., Martin, D. D. E., Bavdaz, M., et al. 1997, A\&AS, 122, 309

Perri, M., Massaro, E., Giommi, P., et al. 2003, A\&A, 407, 453

Pian, E., Vacanti, G., Tagliaferri, G., et al. 1998, ApJ, 492, L17

Piranomonte, S., Giommi, P., Verrecchia, F., \& Perri, M. 2002, in Blazar Astrophysics with BeppoSAX and other Observatories, ed. P. Giommi, E. Massaro, \& G. Palumbo, ASI Special Publ., 51

Quinn, J., Akerlof, C. W., Biller, S., et al. 1996, ApJ, 456, L83

Sambruna, R. M., Aharonian, F. A., Krawczynski, H., et al. 2000, ApJ, 538,127

Tavecchio, F., Maraschi, L., Pian, E., et al. 2001, ApJ, 554, 725

Urry, C. M., Scarpa, R., O'Dowd, M., et al. 2000, ApJ, 532, 816

in 't Zand, J. J. M. 1992, Ph.D. Thesis, Utrecht University 


\section{Online Material}


E. Massaro et al.: The log-parabolic X-ray spectra of Mkn 501, Online Material p 2

Table 1. BeppoSAX NFI observation log of Mkn 501.

\begin{tabular}{lcccccc}
\hline \hline Date & Start UT & End UT & $\begin{array}{c}\text { LECS exposure } \\
(\mathrm{s})\end{array}$ & $\begin{array}{c}\text { MECS exposure } \\
(\mathrm{s})\end{array}$ & $\begin{array}{c}\text { PDS exposure } \\
(\mathrm{s})\end{array}$ & $\begin{array}{c}\text { PDS count rate }^{1} \\
\text { (cts/s) }\end{array}$ \\
\hline $1997 / 04 / 07$ & $05: 11$ & $16: 02$ & 12955 & 20665 & 8936 & $1.94 \pm 0.07$ \\
$1997 / 04 / 11$ & $05: 22$ & $16: 26$ & 13059 & 20430 & 8720 & $2.35 \pm 0.07$ \\
$1997 / 04 / 16$ & $03: 20$ & $14: 36$ & 9812 & 17124 & 7347 & $7.66 \pm 0.08$ \\
& & & & & & \\
$1998 / 04 / 28-29$ & $08: 52$ & $02: 19$ & 13790 & 21911 & 9873 & $1.50 \pm 0.06$ \\
$1998 / 04 / 29-30$ & $20: 33$ & $13: 40$ & 14917 & 21426 & 9662 & $1.80 \pm 0.06$ \\
$1998 / 05 / 01-02$ & $19: 02$ & $10: 38$ & 13402 & 19045 & 8447 & $0.90 \pm 0.07$ \\
$1998 / 06 / 20-21$ & $10: 33$ & $03: 57$ & 17003 & 25871 & 11566 & $0.43 \pm 0.04$ \\
$1998 / 06 / 29-30$ & $19: 41$ & $12: 48$ & 12939 & 18943 & 7471 & $0.52 \pm 0.07$ \\
$1998 / 07 / 16-17$ & $19: 38$ & $11: 06$ & 11443 & 15942 & 6918 & $0.63 \pm 0.07$ \\
$1998 / 07 / 25-26$ & $15: 19$ & $16: 52$ & 25531 & 30952 & 14505 & $0.40 \pm 0.05$ \\
& & & & & & 78415 \\
$1999 / 06 / 10-16$ & $23: 01$ & $02: 11$ & 105849 & 175396 & $0.10 \pm 0.02$ \\
\hline
\end{tabular}

${ }^{1} 15-90 \mathrm{keV}$ energy band. 
E. Massaro et al.: The log-parabolic X-ray spectra of Mkn 501, Online Material p 3

Table 2. BeppoSAX WFC observation log.

\begin{tabular}{|c|c|c|c|c|}
\hline Date & $\begin{array}{l}\text { Exp. } \\
\text { (s) }\end{array}$ & $\begin{array}{r}\text { Offset } \\
\text { (deg) }\end{array}$ & $\begin{array}{c}\text { Count rate }^{1} \\
(\mathrm{cts} / \mathrm{s})\end{array}$ & $\begin{array}{l}\begin{array}{l}\text { Flux } \\
(2 g s)\end{array} \\
(\operatorname{cgs})\end{array}$ \\
\hline $1996 / 09 / 19$ & 67352 & 12.4 & $0.92 \pm 0.10$ & $0.95 \pm 0.10$ \\
\hline 1997/01/04 & 11994 & 13.7 & $1.78 \pm 0.21$ & $1.82 \pm 0.21$ \\
\hline 1997/01/06 & 18496 & 9.3 & $2.28 \pm 0.13$ & $2.34 \pm 0.13$ \\
\hline $1997 / 01 / 11$ & 19713 & 6.0 & $1.65 \pm 0.09$ & $1.69 \pm 0.09$ \\
\hline $1997 / 01 / 27$ & 16370 & 17.0 & $1.78 \pm 0.23$ & $1.82 \pm 0.24$ \\
\hline $1997 / 01 / 29$ & 34958 & 8.6 & $2.10 \pm 0.09$ & $2.15 \pm 0.09$ \\
\hline $1997 / 02 / 22$ & 9621 & 14.4 & $2.17 \pm 0.27$ & $2.22 \pm 0.27$ \\
\hline $1997 / 03 / 02$ & 18414 & 1.8 & $2.35 \pm 0.09$ & $2.41 \pm 0.09$ \\
\hline $1997 / 03 / 04$ & 12226 & 13.4 & $1.96 \pm 0.21$ & $2.01 \pm 0.22$ \\
\hline $1997 / 03 / 05$ & 25846 & 10.4 & $2.31 \pm 0.13$ & $2.37 \pm 0.13$ \\
\hline $1997 / 03 / 15$ & 28148 & 12.5 & $2.42 \pm 0.16$ & $2.48 \pm 0.16$ \\
\hline $1997 / 03 / 26$ & 24729 & 17.4 & $2.77 \pm 0.25$ & $2.84 \pm 0.26$ \\
\hline $1997 / 04 / 01$ & 11490 & 17.1 & $2.87 \pm 0.26$ & $2.94 \pm 0.27$ \\
\hline $1997 / 08 / 30$ & 51124 & 18.5 & $2.34 \pm 0.25$ & $2.39 \pm 0.25$ \\
\hline $1997 / 09 / 04$ & 11342 & 4.5 & $2.51 \pm 0.13$ & $2.57 \pm 0.13$ \\
\hline 1997/09/06 & 11991 & 2.9 & $2.37 \pm 0.11$ & $2.42 \pm 0.11$ \\
\hline 1997/10/01 & 17069 & 2.9 & $2.52 \pm 0.10$ & $2.58 \pm 0.10$ \\
\hline 1997/10/02 & 11121 & 4.5 & $3.16 \pm 0.13$ & $3.24 \pm 0.14$ \\
\hline $1998 / 02 / 02$ & 8256 & 12.4 & $1.50 \pm 0.20$ & $1.53 \pm 0.20$ \\
\hline $1998 / 02 / 24$ & 28014 & 3.9 & $1.13 \pm 0.08$ & $1.16 \pm 0.08$ \\
\hline $1998 / 02 / 28$ & 12388 & 3.9 & $1.71 \pm 0.10$ & $1.75 \pm 0.10$ \\
\hline $1998 / 03 / 08$ & 35681 & 13.1 & $1.55 \pm 0.17$ & $1.59 \pm 0.17$ \\
\hline $1998 / 07 / 02$ & 25965 & 9.0 & $1.02 \pm 0.13$ & $1.05 \pm 0.14$ \\
\hline $1998 / 07 / 29$ & 96891 & 13.3 & $1.53 \pm 0.10$ & $1.56 \pm 0.10$ \\
\hline $1998 / 08 / 06$ & 34447 & 3.6 & $1.44 \pm 0.07$ & $1.48 \pm 0.07$ \\
\hline 1998/08/11 & 27383 & 4.2 & $1.19 \pm 0.08$ & $1.22 \pm 0.08$ \\
\hline $1998 / 08 / 24$ & 19363 & 6.2 & $1.47 \pm 0.08$ & $1.51 \pm 0.08$ \\
\hline $1998 / 09 / 03$ & 11044 & 8.5 & $0.95 \pm 0.14$ & $0.97 \pm 0.15$ \\
\hline 1998/09/04 & 18926 & 8.5 & $1.50 \pm 0.13$ & $1.53 \pm 0.13$ \\
\hline 1998/09/16 & 24681 & 3.6 & $1.22 \pm 0.06$ & $1.25 \pm 0.07$ \\
\hline $1998 / 09 / 17$ & 14362 & 6.2 & $1.61 \pm 0.14$ & $1.65 \pm 0.14$ \\
\hline $1998 / 10 / 07$ & 18958 & 12.4 & $1.22 \pm 0.16$ & $1.25 \pm 0.17$ \\
\hline $1998 / 10 / 27$ & 16099 & 9.2 & $1.18 \pm 0.11$ & $1.20 \pm 0.11$ \\
\hline $1999 / 02 / 07$ & 55780 & 19.9 & $1.92 \pm 0.25$ & $1.96 \pm 0.26$ \\
\hline $1999 / 02 / 27$ & 22592 & 7.5 & $2.11 \pm 0.10$ & $2.16 \pm 0.10$ \\
\hline $1999 / 03 / 14$ & 61290 & 12.2 & $1.23 \pm 0.10$ & $1.26 \pm 0.10$ \\
\hline $1999 / 03 / 29$ & 74910 & 6.6 & $1.05 \pm 0.07$ & $1.08 \pm 0.07$ \\
\hline $1999 / 03 / 31$ & 50662 & 10.4 & $1.02 \pm 0.07$ & $1.05 \pm 0.07$ \\
\hline $1999 / 04 / 05$ & 30384 & 16.6 & $1.41 \pm 0.16$ & $1.45 \pm 0.16$ \\
\hline $1999 / 05 / 01$ & 53604 & 14.3 & $0.81 \pm 0.12$ & $0.83 \pm 0.12$ \\
\hline $1999 / 08 / 08$ & 62916 & 3.6 & $0.41 \pm 0.05$ & $0.42 \pm 0.05$ \\
\hline $1999 / 08 / 13$ & 18946 & 2.9 & $0.81 \pm 0.10$ & $0.83 \pm 0.10$ \\
\hline $1999 / 08 / 28$ & 61318 & 13.7 & $1.08 \pm 0.17$ & $1.10 \pm 0.18$ \\
\hline 1999/09/11 & 23178 & 6.1 & $0.95 \pm 0.10$ & $0.97 \pm 0.10$ \\
\hline $1999 / 09 / 24$ & 15640 & 8.9 & $1.09 \pm 0.13$ & $1.12 \pm 0.13$ \\
\hline $2000 / 02 / 15$ & 32282 & 3.9 & $0.46 \pm 0.07$ & $0.47 \pm 0.07$ \\
\hline $2000 / 03 / 05$ & 24423 & 13.3 & $0.92 \pm 0.13$ & $0.95 \pm 0.13$ \\
\hline $2000 / 03 / 08$ & 23440 & 13.3 & $0.90 \pm 0.14$ & $0.92 \pm 0.14$ \\
\hline $2000 / 03 / 10$ & 39579 & 13.9 & $1.15 \pm 0.11$ & $1.18 \pm 0.12$ \\
\hline $2000 / 03 / 12$ & 43738 & 4.0 & $0.74 \pm 0.07$ & $0.76 \pm 0.08$ \\
\hline $2000 / 03 / 13$ & 22417 & 4.0 & $0.76 \pm 0.10$ & $0.77 \pm 0.10$ \\
\hline
\end{tabular}

Table 2. continued.

\begin{tabular}{lrrcc}
\hline \hline Date & $\begin{array}{c}\text { Exp. } \\
(\mathrm{s})\end{array}$ & $\begin{array}{r}\text { Offset } \\
\text { (deg) }\end{array}$ & $\begin{array}{c}\text { Count rate }^{1} \\
\text { (cts/s) }\end{array}$ & $\begin{array}{c}\text { Flux }^{2} \\
(\mathrm{cgs})\end{array}$ \\
\hline $2000 / 03 / 19$ & 22013 & 2.6 & $0.69 \pm 0.09$ & $0.70 \pm 0.09$ \\
$2000 / 03 / 20$ & 31737 & 10.4 & $0.60 \pm 0.11$ & $0.62 \pm 0.12$ \\
$2000 / 08 / 01$ & 36511 & 7.4 & $0.81 \pm 0.10$ & $0.83 \pm 0.10$ \\
$2000 / 08 / 17$ & 85411 & 4.5 & $0.42 \pm 0.05$ & $0.43 \pm 0.05$ \\
$2000 / 08 / 20$ & 33638 & 6.9 & $0.66 \pm 0.09$ & $0.67 \pm 0.10$ \\
$2000 / 08 / 21$ & 28547 & 11.1 & $0.62 \pm 0.10$ & $0.63 \pm 0.11$ \\
$2000 / 09 / 05$ & 46527 & 3.6 & $0.63 \pm 0.06$ & $0.65 \pm 0.06$ \\
$2000 / 09 / 10$ & 37925 & 2.9 & $0.59 \pm 0.07$ & $0.60 \pm 0.07$ \\
$2000 / 09 / 23$ & 39293 & 7.6 & $0.67 \pm 0.09$ & $0.69 \pm 0.09$ \\
$2000 / 10 / 03$ & 37820 & 6.9 & $0.91 \pm 0.08$ & $0.93 \pm 0.08$ \\
$2001 / 02 / 11$ & 27294 & 11.1 & $0.76 \pm 0.13$ & $0.77 \pm 0.13$ \\
$2001 / 02 / 16$ & 30427 & 12.4 & $0.76 \pm 0.11$ & $0.77 \pm 0.11$ \\
$2001 / 02 / 25$ & 33444 & 2.6 & $0.64 \pm 0.07$ & $0.66 \pm 0.07$ \\
$2001 / 08 / 09$ & 37884 & 4.5 & $0.55 \pm 0.07$ & $0.56 \pm 0.07$ \\
$2001 / 08 / 14$ & 81153 & 8.6 & $0.63 \pm 0.07$ & $0.65 \pm 0.08$ \\
$2001 / 08 / 17$ & 120472 & 10.4 & $0.82 \pm 0.08$ & $0.85 \pm 0.08$ \\
$2001 / 08 / 22$ & 56668 & 10.0 & $0.63 \pm 0.09$ & $0.65 \pm 0.09$ \\
$2001 / 09 / 11$ & 37706 & 0.3 & $0.71 \pm 0.07$ & $0.73 \pm 0.07$ \\
$2001 / 09 / 21$ & 58224 & 0.3 & $0.55 \pm 0.05$ & $0.56 \pm 0.05$ \\
$2001 / 10 / 03$ & 23808 & 0.3 & $0.64 \pm 0.07$ & $0.66 \pm 0.07$ \\
\hline
\end{tabular}

${ }^{1} 2-8 \mathrm{keV}$ energy band, i.e. the band of maximum sensitivity for the two instruments.

$2-10 \mathrm{keV}$ energy band, in units of $10^{-10} \mathrm{erg} \mathrm{cm}^{-2} \mathrm{~s}^{-1}$. 